\title{
La enseñanza en la Universidad Pedagógica Nacional desde las creencias de sus docentes
}

\section{Education in the National Pedagogical University from the teachers' beliefs}

José Simón Sánchez Hernández

Alejandra Castillo Peña

Universidad Pedagógica Nacional (UPN)

\author{
María del Carmen Farfán García \\ Enrique Navarrete Sánchez \\ Universidad Autónoma del \\ Estado de México (UAEMéx)
}

\section{Resumen}

La finalidad de este trabajo es describir los resultados obtenidos en las dimensiones denominadas: Creencias, Propósitos y Acciones del Inventario de las Perspectivas de Enseñanza (IPE) en una muestra de profesores de la Universidad Pedagógica Nacional (UPN) Ajusco, así como identificar las características de formación y profesionalización de los docentes. Para tal efecto, se aplicó el instrumento versión en español modificada del Teaching Perspectives Inventory (TPI), a 27 docentes de la UPN, Ajusco. Los resultados obtenidos indican que los docentes tienen una idea clara de su función en la enseñanza y la experiencia suficiente para facilitar la forma de enseñar el contenido, y los resultados que se esperan en el aprendizaje de los estudiantes. Para mejorar la calidad de la educación, es importante comprender la forma de enseñar, ya que de esta manera se pueden explicar las acciones que el docente lleva a cabo en el salón de clases. Asimismo, se da la posibilidad de considerar a las creencias docentes como parte de la modificación de los contenidos curriculares y/o en la formación permanente del docente.

Palabras clave: enseñanza superior, creencias, docente, formación.

Nota del autor

José Simón Sánchez Hernández Universidad Pedagógica Nacional (UPN). Alejandra Castillo Peña Universidad Pedagógica Nacional (UPN). María del Carmen Farfán Facultad de Ciencias de la Conducta de la Universidad Autónoma del Estado de México (UAEMéx). Enrique Navarrete Sánchez Facultad de Ciencias de la Conducta de la Universidad Autónoma del Estado de México (UAEMéx). La correspondencia en relación con este artículo debe dirigirse a José Simón Sánchez Hernández Universidad Pedagógica Nacional (UPN), Carr. Picacho Ajusco 24, Equipamiento Periférico Picacho Ajusco Canal 13, 14200 Ciudad de México, CDMX.

Dirección electrónica: simsanher@yahoo.com Tel.: 5527450291 


\begin{abstract}
The purpose of this paper is to describe the results obtained in the Inventory of Teaching Perspectives (IPE) in its three dimensions Beliefs, Purposes and Actions in a sample of teachers from the National Pedagogical University (UPN) Ajusco, as well as to identify the characteristics of training and professionalization of them. For that purpose, a modified Spanish version of the Teaching Perspectives Inventory, from Pratt and Collins (2001) was applied to 27 teachers from UPN Ajusco. The results obtained indicate that teachers have a clear idea of their role in education and the experience to facilitate the teaching process of the content, as well as the results that are expected in the student's learning. In order to improve the quality of education, it is important to understand the form in which the students are educate, therefore, it can explain the actions the teacher carries out in the classroom. Likewise, it is possible to consider teacher beliefs as part of the modification of the curricular contents and / or in the permanent formation of the teacher.
\end{abstract}

Keywords: higher education, beliefs, teacher, formation.

Ante los cambios pedagógicos en los actuales modelos educativos, el profesor transforma la manera de enseñar: pasa de un entorno donde él como especialista era el centro del proceso, a otro donde debe centrarse en el aprendizaje y plantear situaciones reales del entorno de sus alumnos para que ellos puedan poner en práctica los conocimientos.

Esto implica que los estudiantes aprendan significativamente, se motiven y puedan ver la aplicabilidad casi inmediata del conocimiento que adquieren en el aula. Por lo tanto, el docente les ayuda a asumir la responsabilidad de su propio aprendizaje y aprender a aprender en el contexto de la nueva cultura del aprendizaje (Pozo \& Pérez, 2009). Esta transformación no es fácil y menos cuando el docente se formó en un modelo donde le enseñaron a impartir contenidos de una forma diferente y su modelo de referencia son las clases magistrales, basadas en el discurso. En los llamados nuevos modelos educativos, los planes y programas se reorientan al cambio de contenidos, sin embargo, no se pone atención a la forma en que se manejan esos contenidos, de tal manera que el alumno pueda encontrar el sentido fundamental de su conocimiento que, como ya se mencionó, es la aplicabilidad del mismo o saber hacer. Así que, al modificar los planes y programas de estudio, nos preguntamos si previamente se considera el cambio o transformación en las concepciones de los profesores en el proceso de enseñanzaaprendizaje, ya que de esta manera se modificaría la forma de impartir la clase acorde con los requerimientos actuales. La idea central es entonces, la importancia de la enseñanza como uno de los ejes fundamentales del proceso educativo. Peralta (2015) menciona que “... organizar el proceso de enseñanza en un mundo con los cambios actuales, en un mundo globalizado, 
es una labor del educador que a veces crea incertidumbre e insatisfacción" (p. 1).

Por su parte, Vaillant (2007) considera que el discurso de los maestros en relación a los factores que generan insatisfacción con la tarea se asemeja a un amplio listado de quejas. Dicho autor afirma que se manifiesta un proceso de descomposición de un modelo clásico de enseñanza y de ejercicio de la profesión, sin que otro modelo alternativo haya emergido hasta ahora, donde una amplia mayoría de maestros describe un modelo de profesionalidad en plena alteración, sin que aparezca otro lo suficientemente pertinente y consistente para tomar el relevo.

Vale la pena decir que el docente en los modelos educativos actuales recupera un papel fundamental en el proceso de enseñanzaaprendizaje. Por esta razón, para la nueva visión en torno a la enseñanza, el profesor debe crear ambientes de aprendizaje propicios para el desarrollo de los contenidos de su unidad, ofrecer más y mejores oportunidades de aprendizaje a los estudiantes. Para Rodríguez, Suárez y González-Permuy (2005), la enseñanza en este caso forma parte intrínseca y plena del proceso educativo y posee como su núcleo básico el aprendizaje. En efecto, la enseñanza, incluido el aprendizaje, constituye en el contexto escolar un proceso de interacción e intercomunicación entre varios sujetos y fundamentalmente tiene lugar en forma grupal. En dicho grupo, el maestro ocupa un lugar de gran importancia como pedagogo, lo organiza y lo conduce, pero tiene que ser de tal manera que los miembros de ese grupo (alumnos) tengan un significativo protagonismo y sientan una gran motivación por lo que hacen (Rodríguez et al., 2005).

Para Contreras (1990, citado en González, 2003), enseñar es "provocar dinámicas y situaciones en las que pueda darse el proceso de aprender en los alumnos" (pág. 2), de acuerdo con esta autora, una de las características esenciales de la enseñanza es la intencionalidad. González (2003), menciona que los alumnos adquieren muchos conocimientos fuera del salón de clases de manera cotidiana, pero es en el salón de clases donde realmente aprenden de manera intencional lo que el profesor quiere enseñarles. En otras palabras, es necesario recordar que el profesor cuando enseña tiene una intención sobre el aprendizaje del alumno, misma que le permite poner en práctica una serie de estrategias para facilitar el aprendizaje. Autores como Hargreaves (2005) y Zabalza (2009) mencionan que hoy en día la enseñanza superior debe implementar nuevas maneras de educar y abordar las relaciones que se establecen dentro del aula, pensar en el desarrollo y la innovación; potenciar habilidades cognitivas, sociales, afectivas y comunicativas de sus alumnos; hacerse cargo de formar a sujetos participativos, creativos, proactivos, etc., con el fin de responder a las expectativas que la sociedad ha formulado, a partir de una nueva realidad más compleja y globalizada.

Por otro lado, estudios internacionales como los realizados en la Universidad de Barcelona 
o en la Universidad de la Habana, coinciden en señalar que una de las claves para mejorar la calidad de la educación es mejorar las competencias profesionales de los docentes a través de actividades de formación que contemplen la adecuación de los conocimientos y métodos a la evolución de las ciencias y de las didácticas específicas. Así mismo, es importante que la formación permanente no sólo asegure espacios de aprendizaje de forma tradicional, sino también ambientes diversificados, donde el trabajo permita una revisión de las representaciones del profesor (creencias, conceptos, roles, sentimientos, etc.), en pos de trabajar sobre una redescripción representacional de la realidad(es) para desaprender y aprender en el ejercicio profesional atendiendo al contexto (Karmiloff-Smith, 1994).

En relación con la formación profesional y la enseñanza Contreras (2014), señala que la calidad de un programa formativo depende, en lo fundamental, de las condiciones de enseñanza a las que se ven expuestos los futuros profesionales, derivándose de esto que las habilidades e intervenciones pedagógicas de los profesores cobren especial relevancia.

De acuerdo con Peralta (2015), en el momento actual, cuando hablamos del proceso enseñanza y de aprendizaje, se aprecia una preocupación por lo que se ha de enseñar, y no solo eso, sino, cómo se va a enseñar, cómo van los estudiantes a desarrollar habilidades que les permitan dar continuidad al proceso. De este modo, se habla, entonces, de la estrategia que es utilizada como un medio o un recurso de la enseñanza que responde a la manera de enseñar. Pimienta (2012), define las estrategias como “... instrumentos de los que se vale un docente para contribuir a la implementación y desarrollo de las competencias de los estudiantes" (p. 3).

Brevemente, cuando el docente enseña, a su vez integra objetivos, contenidos y actividades en función de los conocimientos y/o habilidades que debe poseer el alumno para facilitar el proceso de aprendizaje. Es así como cada docente define su propio estilo de enseñanza, determinado por las creencias, la intencionalidad educativa y la determinación de los objetivos, así como las actividades y estrategias empleadas para lograrlos. En consecuencia, al enseñar, el profesor cree, tiene una intención y actúa para propiciar el aprendizaje en sus alumnos. Todo esto para referir el papel que tienen las creencias en la enseñanza. Fickel (1999, citado en Jiménez \& Feliciano, 2006), las define como "un conjunto de potentes esquemas sobre la educación, el conocimiento, el aprendizaje y la enseñanza, a partir de las cuales los docentes toman decisiones conscientes y priorizadas" (p. 109). También se las consigna como juicios personales que predicen formas de comportamiento y que guían los pensamientos de las personas (Gómez, 2010).

Al respecto, Pratt y Collins (2001) amplían las creencias de los profesores acerca de la enseñanza, ya que establecieron el concepto de "perspectivas de enseñanza" (teaching perspectives), que definieron como "un conjunto 
interrelacionado de creencias e intenciones que da dirección y justificación a nuestras acciones". Así, la perspectiva es un término más amplio que las creencias, pues integra en su significación aspectos que se relacionan con las creencias, los propósitos y las acciones de los profesores cuando enseñan. De esta manera, cada perspectiva puede ser observada desde tres dimensiones: Creencias, Intenciones y Acciones que los profesores realizan cuando enseñan. De acuerdo con estos autores, el componente Creencia se refiere a lo que el profesor cree acerca de la enseñanza; el componente Acción, a lo que hacen los profesores cuando enseñan, y el componente Intención, a lo que éstos pretenden lograr cuando enseñan.

Es por esto que, dada la importancia de las creencias docentes para comprender la forma enseñanza, se realizó la presente investigación, con el fin de explicar las acciones que el docente lleva a cabo en el salón de clases. De esta manera, se recupera la importancia de las creencias, de tal forma que sean consideradas en el momento de modificar los contenidos curriculares y/o en la formación permanente del docente.

\section{Método}

La presente investigación corresponde a una metodología cuantitativa de tipo descriptivo, cuyo objetivo general se establece como describir los resultados obtenidos en las dimensiones del IPE de una muestra de 27 profesores de la UPN, Ajusco. Adicionalmente, se espera identificar las características de formación y perspectivas de enseñanza de una muestra de docentes de la UPN.

\section{Instrumento}

La instrumentación de la investigación fue a través del mencionado IPE. Este cuestionario fue desarrollado inicialmente con el fin de identificar perfiles de los profesores con respecto a cinco perspectivas acerca de la enseñanza. El instrumento pretende identificar qué es lo que creen, se proponen lograr y hacen los profesores cuando enseñan. Originalmente, fue elaborado en inglés con el nombre ya antes enunciado. Está conformado por 45 reactivos con una categoría de respuestas que va de 1 al 5, subdividido en cinco perspectivas: Transmisión, Aprendizaje, Desarrollo, Acompañamiento y Reforma social, con nueve reactivos cada una. A su vez, cada perspectiva puede ser observada desde tres dimensiones: Creencias, Intenciones $\mathrm{o}$ Propósitos y Acciones que los profesores realizan cuando enseñan. Cada una de las dimensiones se responde de acuerdo con una escala de cinco puntos. Las Creencias, con una categoría de respuesta que va de 1 (Completamente en desacuerdo) a 5 (Completamente de acuerdo). Mientras que las dimensiones Propósitos y Acciones emplean una categoría de cinco puntos que va de 1 (Nunca) a 5 (Siempre).

\section{Análisis de datos}

Para el análisis de los resultados se utilizó la estadística descriptiva, determinando con ello los porcentajes, frecuencias y medias en cada uno 
de los aspectos del IPE, para la interpretación de los resultados con respecto a las tres dimensiones se analizaron los porcentajes por cada reactivo.

Una vez recopilada la información de los 27 docentes, se realizó el análisis estadístico correspondiente. En primera instancia, se obtuvieron los resultados en forma de porcentajes sobre la formación y profesionalización docente, posteriormente, se obtuvo la media para cada una de las dimensiones: Creencias (C), Propósitos (P) y Acciones (A), con base en las respuestas de los participantes; el mínimo puntaje por cada dimensión es 3 y máximo 15. Más tarde se obtuvo la media general de las tres dimensiones: 3.7.

\section{Resultados}

\section{Función principal en la institución}

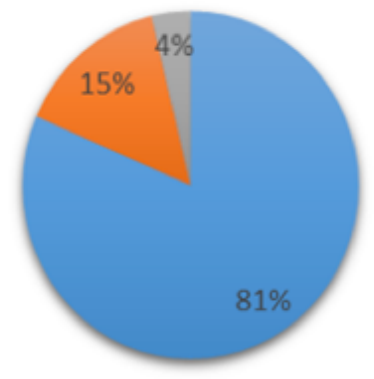

घ Docencia - Investigación U Gestión

Figura 1. Porcentajes de función principal en la institución
En la figura 1 se puede observar la función principal de los docentes en la institución, donde el $81.48 \%$ de los docentes se dedica principalmente a la docencia dentro de la UPN, Ajusco; el 14.81\% a la investigación; y el 3.70\% a gestión.

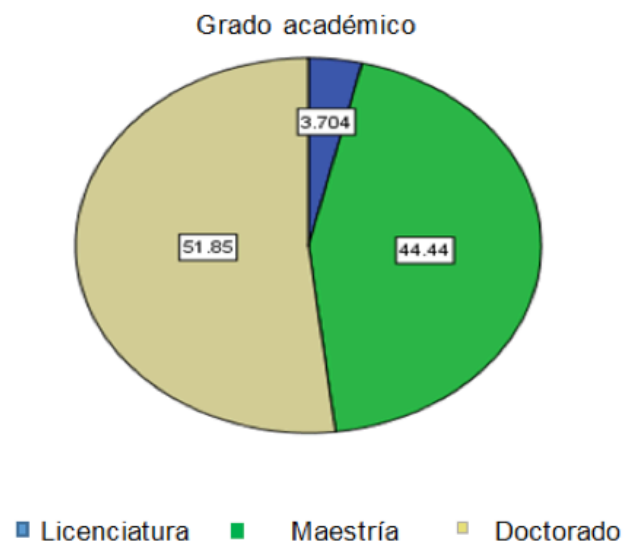

Figura 2. Porcentajes de grado académico

En la figura 2, se puede distinguir que el grado académico de los docentes, donde el $51.85 \%$ tiene Doctorado, 44.44\% Maestría y el $3.70 \%$ Licenciatura.

En la figura 3, se muestra el área de especialización de acuerdo con el grado de estudios más alto de los docentes; los porcentajes más altos fueron obtenidos en el área de Psicología con 48.15\%, posteriormente Educación/Pedagogía con 25.93\%, seguido por Sociales y Comunicaciones con $7.4 \%$; y el resto se distribuye en porcentajes similares en las áreas de Ingeniería, Sociales, Lenguas, Matemáticas con 3.7\%. 
La figura 4 presenta los años dedicados a la docencia que tienen los participantes en la investigación, los resultados indican que el $40.74 \%$ tiene 25 años o más; el 33\% tiene de 9 a
$16 ; 14.81 \%$ se encuentra en el rango de 17 a 24 años; y por último, el $11.11 \%$ tiene 1 a 8 años dedicados a la docencia.

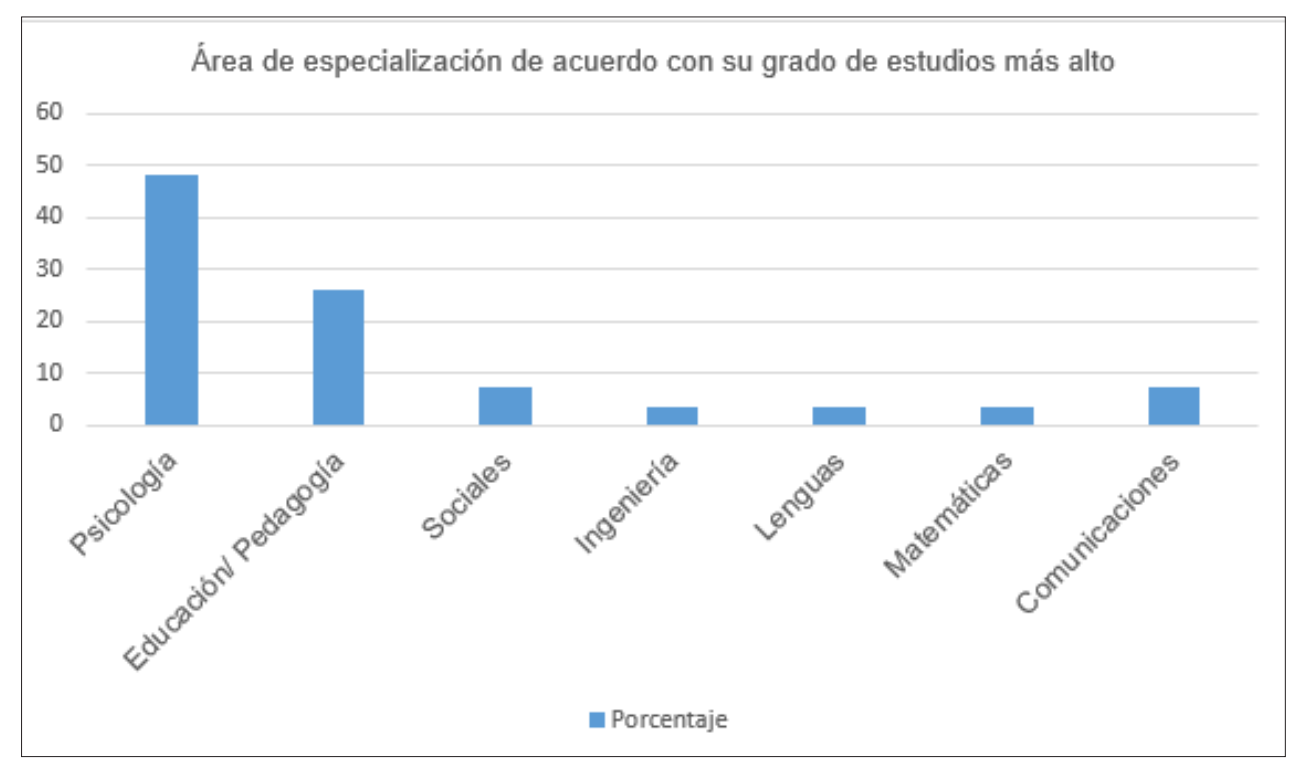

Figura 3. Porcentajes de participantes por área de especialización de acuerdo con su grado de estudios más alto de los docentes

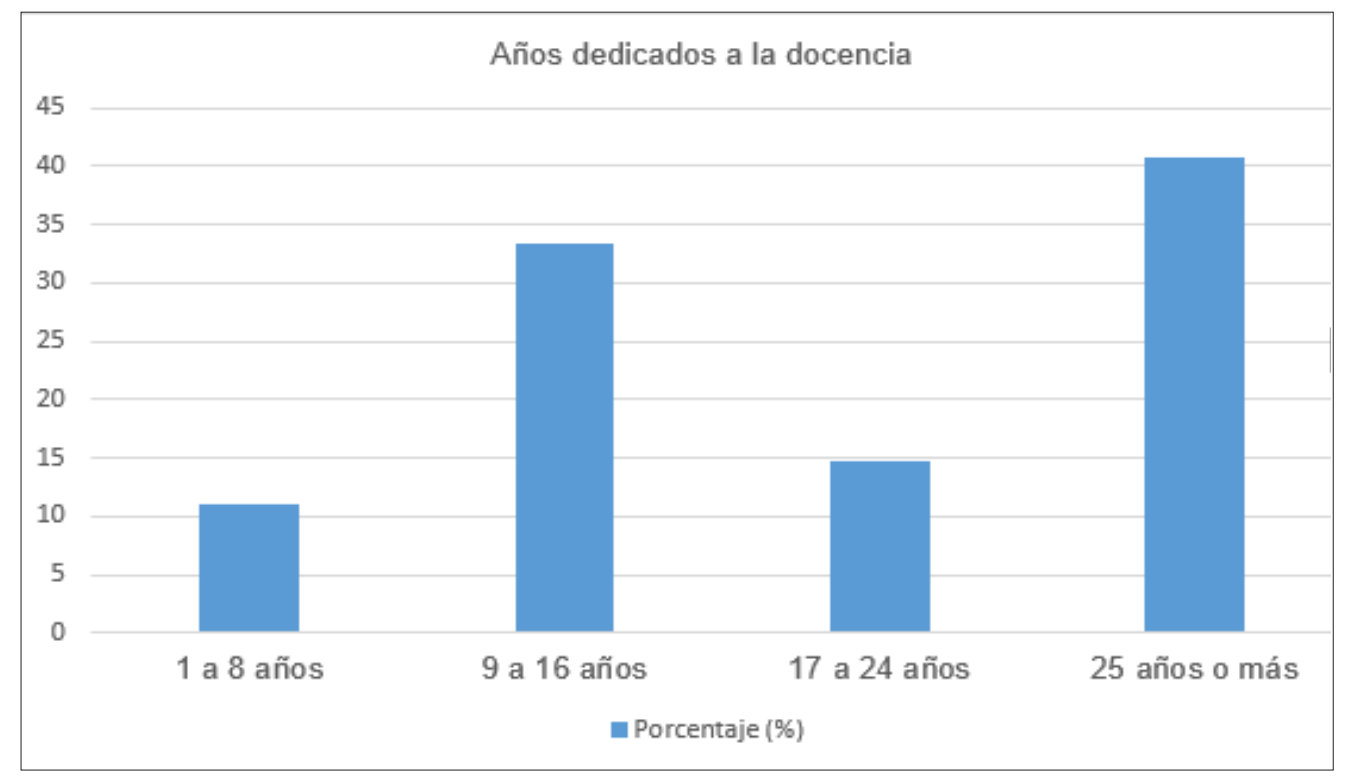

Figura 4. Porcentaje de años dedicados a la docencia 
Tabla 1

Medias de las dimensiones del inventario de las perspectivas de enseñanza

\begin{tabular}{lcc}
\hline Dimensiones & Media & D.E. \\
\hline Creencias & 3.68 & .478 \\
Propósitos & 3.80 & .675 \\
Acciones & 3.81 & .579 \\
Total & 3.76 & .072 \\
\hline
\end{tabular}

En lo que respecta a los resultados obtenidos con la aplicación del instrumento, en la tabla 1 se observa que las tres dimensiones obtienen de manera general una media de 3.76 con una D.E. de .072, y de manera particular las Creencias tienen una media de 3.68 con D.E. de .478; los Propósitos, una media de 3.80 con una D.E. de .675; y las Acciones, una media de 3.81 y una D.E. de .579 .

\section{Discusión}

De acuerdo con los resultados, se tiene una media total en las tres dimensiones del instrumento de 3.76; resulta interesante mencionar que existe relación entre las dimensiones. Según Pratt, Boll y Collins (2007), para que la enseñanza sea realmente efectiva, se requiere armonía entre las estrategias empleadas para enseñar y las Creencias, Intenciones o Propósitos y Acciones de los profesores. De manera específica y diferente a la interpretación del instrumento tradicional, centrada en las perspectivas, realizamos la interpretación por dimensión, para lo cual se revisaron las medias de cada respuesta.
En relación con las Creencias, el resultado nos lleva a interpretar que, para el docente, la enseñanza debe enfocarse hacia el desarrollo de cambios cualitativos de pensamiento; el aprendizaje se beneficia cuando hay objetivos predeterminados y debe tener dominio completo de su materia. Si hace un acercamiento a los resultados de formación, esto coincide con que los docentes participantes en la investigación tienen estudios de posgrado, lo cual implica una preparación más especializada de su disciplina.

Con respecto a los Propósitos, en la enseñanza, los resultados nos indican que los docentes esperan que las personas desarrollen nuevas maneras de pensar el contenido de la materia, además, esperan que las personas apliquen el contenido de la materia en la vida real, lo que corresponde al sentido de los modelos educativos actuales. Así también, las Acciones de los docentes se encaminan a relacionar el contenido con aplicaciones o escenarios de la vida real; hacen muchas preguntas mientras enseñan, especifican lo que se debe de aprender y ayudan a que las personas vean la necesidad de cambios en la sociedad.

En lo que atañe a las características de formación y profesionalización, existe una coherencia entre los mismos y los resultados obtenidos con el instrumento, ya que los docentes tienen una amplia experiencia en la docencia y un alto grado de estudios, pueden ser especialistas, fundamentalmente en el área de Psicología y Educación. 
Es importante reconocer que los resultados tienen una relación directa con la Misión y los principios generales de la UPN. En el caso particular de la Misión, se señala que se orienta a la formación y desarrollo de profesionales de la educación y a la generación de conocimiento de acuerdo con las necesidades del país, considerando la diversidad sociocultural. Esto es factible de lograr, cuando los docentes cuentan con un alto grado académico que les permite no solo conocer su disciplina, sino también manejar los contenidos actuales acorde con los modelos educativos. Según la interpretación de los resultados obtenidos, el docente tiene una idea clara y la experiencia suficiente que le permite facilitar los contenidos a enseñar, cómo hacerlo y los resultados que se esperan en el aprendizaje de los estudiantes. Además, desarrollan principalmente la función de docencia, seguida de la investigación, lo cual les permite articular la generación del conocimiento con los contenidos, con base en el desarrollo y la innovación pedagógica.

Finalmente, las creencias docentes que definen el estilo de enseñanza de los participantes, tienen estrecha relación con la institución y con el modelo educativo actual, de alguna manera esto garantiza que se trabaja para mejorar la calidad de la educación, al adecuar los conocimientos y métodos a la evolución de las ciencias y de las didácticas específicas. No obstante, los resultados encontrados, sería conveniente promover procesos de reflexión y redescripción sobre estas perspectivas de enseñanza entre los docentes, para saber más del significado e influencia específica de estas perspectivas en el aprendizaje de los estudiantes.

\section{Referencias}

Contreras, C. (2014). El desarrollo docente del formador de profesores: una propuesta orientada hacia el análisis de incidentes críticos auténticos. Estudios Pedagógicos, X(1), número especial, 49-69. Recuperado de https://scielo.conicyt.cl/pdf/estped/v40nEspecial/art04.pdf

Gómez, V. (2010). Explorando el cambio epistemológico y conceptual en la Formación Inicial de Profesores en distintos contextos universitarios. Informe final proyectos FONIDE 2009. Documento inédito.

González, V. (2003). Estrategias de enseñanza y aprendizaje. México: Pax.

Hargreaves, A. (2005). Profesorado, cultura y postmodernidad. Cambian los tiempos, cambia el profesorado. Madrid: Morata.

Jiménez, A., \& Feliciano, L. (2006). Pensar el pensamiento del profesorado. Revista Española de Pedagogía, LXIV(233), 105122. Recuperado de https://dialnet.unirioja. es/servlet/articulo?codigo $=1973270$

Karmiloff-Smith, A. (1994). Más allá de la modularidad: La ciencia cognitiva desde la perspectiva del desarrollo. Madrid: Alianza.

Peralta, W. (2015). El docente frente a las estrategias de enseñanza aprendizaje. Revista Vinculando. Recuperado de http://vinculando.org/educacion/rol-del- 
docente-frente-las-recientes-estrategias-de-

Recibido: 12/07/2017

ensenanza-aprendizaje.html

Revisado: 06/12/2017

Pimienta, J. (2012). Estrategias de enseñanza-

aprendizaje. México: Pearson.

Pozo, I., \& Pérez E. (2009) Psicología del aprendizaje universitario: La formación en competencias. Madrid: Morata.

Pratt, D., \& Collins, J. (2000). The Teaching Perspectives Inventory. In Proceedings of the Forty-First Adult Education Research Conference. Vancouver. (ED 452 417) Recuperado de http://teachingperspectives. com

Pratt, D. D., \& Collins, J. B. (2001). Teaching perspectives inventory. Recuperado de http:// teachingperspectives.com

Pratt, D., Boll, S., \& Collins, J. (2007). Towards a plurality of perspectives for nurse educators. Nursing Philosophy, 8(1), 4959. doi:10.1111/j.1466-769X.2007.00297.x

Rodríguez, J., Suárez, A., González., \& Permuy, L. D. (2005). Acercamiento Necesario a la Pedagogía General. La Habana: Editorial Pueblo y Educación, 28-35.

Vaillant, D. (2007). Nuevas Tendencias en la Formación Permanente del Profesorado. Barcelona: Preal. Recuperado de http://www. ub.edu/obipd/docs/la_identidad_docente vaillant_d.pdf

Zabalza, M. (2009). Ser profesor universitario hoy. La cuestión universitaria, (5), 69-81. Recuperado de polired.upm.es/index.php/ lacuestionuniversitaria/article/download/ $3338 / 3403$ 